\section{(2) \\ BRAZIILIAN JOURNAL \\ OF MEDICAL AND BIOLOGICAL RESEARCH}

www.bjournal.com.br
ISSN 0100-879X

Volume 45 (5) 376-472 May 2012

BIOMEDICAL SCIENCES

AND

CLINICAL INVESTIGATION

Braz J Med Biol Res, May 2012, Volume 45(5) 425-435

doi: 10.1590/S0100-879X2012007500053

Influence of cueing on the preparation and execution of untrained and trained complex motor responses

S.R. Alouche, G.N. Sant'Anna, G. Biagioni and L.E. Ribeiro-do-Valle

The Brazilian Journal of Medical and Biological Research is partially financed by

\section{Q QNPPq}

Ministério

da Ciência e Tecnologia

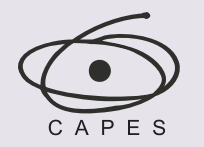

Ministério da Educação

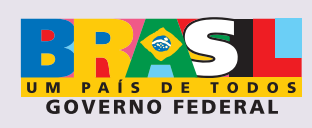

DTFAPESP

Institutional Sponsors

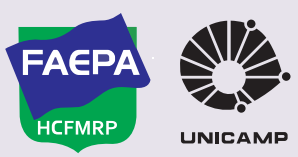

$\oplus$ SHIMADzu

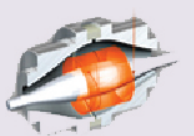

UNICAMP

12DD Associaçäo Explore High - Performance MS Orbitrap Technology In Proteomics \& Metabolomics
SciE/O
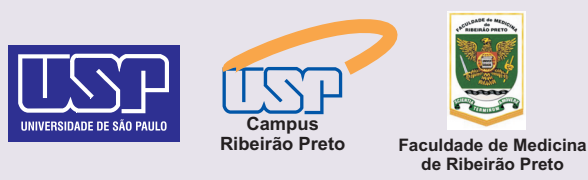
analitica $\underset{\text { analiticaweb.com.br }}{\text { Thermo }}$ S I ENIFIC 


\title{
Influence of cueing on the preparation and execution of untrained and trained complex motor responses
}

\author{
S.R. Alouche ${ }^{1}$, G.N. Sant'Anna², G. Biagioni² and L.E. Ribeiro-do-Valle ${ }^{2}$ \\ 1Departamento de Fisioterapia, Universidade Cidade de São Paulo, São Paulo, SP, Brasil \\ ${ }^{2}$ Departamento de Fisiologia e Biofísica, Instituto de Ciências Biomédicas, \\ Universidade de São Paulo, São Paulo, SP, Brasil
}

\begin{abstract}
This study investigated the influence of cueing on the performance of untrained and trained complex motor responses. Healthy adults responded to a visual target by performing four sequential movements (complex response) or a single movement (simple response) of their middle finger. A visual cue preceded the target by an interval of 300, 1000, or 2000 ms. In Experiment 1 , the complex and simple responses were not previously trained. During the testing session, the complex response pattern varied on a trial-by-trial basis following the indication provided by the visual cue. In Experiment 2, the complex response and the simple response were extensively trained beforehand. During the testing session, the trained complex response pattern was performed in all trials. The latency of the untrained and trained complex responses decreased from the short to the medium and long cue-target intervals. The latency of the complex response was longer than that of the simple response, except in the case of the trained responses and the long cue-target interval. These results suggest that the preparation of untrained complex responses cannot be completed in advance, this being possible, however, for trained complex responses when enough time is available. The duration of the 1st submovement, 1st pause and 2nd submovement of the untrained and the trained complex responses increased from the short to the long cue-target interval, suggesting that there is an increase of online programming of the response possibly related to the degree of certainty about the moment of target appearance.
\end{abstract}

Key words: Cueing; Cue-target interval; Response complexity; Response practice; Reaction time; Movement time

\section{Introduction}

The latency and duration of a complex response is reduced when its exact pattern is previously indicated by a cue. This was demonstrated for key-press responses (1), syllable-articulation responses (2) and aiming responses $(3,4)$. The finding has been attributed to the preparation of the complex response in advance.

Klapp (1) observed that the latency of unfamiliar four-unit key-press responses was longer than the latency of unfamiliar one-unit key-press responses when the response pattern was indicated by a cue well in advance, characterizing a simple reaction time task. These results and similar ones obtained in a subsequent study, in which syllable-articulation responses were evaluated (2), led him to propose that the programming process would involve the organization of the internal structure of the response, in the case of single-unit responses, or of the 1st unit ("chunk") of the response, in the case of multiple-unit responses. In addition, it would involve the activation and scanning of an abstract time frame, which specifies the time of initiation of each unit of the response, independently of its content. The organization of the internal structure of the 1st unit of the response and the ordering of the response units would evolve and could even be completed before the triggering of the response, but the activation and scanning of the abstract time frame would only be possible after that.

Using the same kind of task, Klapp (1) further observed that with practice the latency of the four-unit key-press responses and the latency of the one-unit key-press responses decreased and, most interesting, the latency of the four-unit key-press responses became similar to that of the one-unit key-press responses. He explained this latter finding by arguing that with practice the four-unit key-press

Correspondence: L.E. Ribeiro-do-Valle, Departamento de Fisiologia e Biofísica, ICB, USP, Av. Prof. Lineu Prestes, 1524, 05508-000 São Paulo, SP, Brasil. Fax: +55-11-3091-7364. E-mail: ribeiro@icb.usp.br

Received July 29, 2011. Accepted March 23, 2012. Available online April 5, 2012. Published May 7, 2012. 
responses became in fact one-unit key-press responses due to the central chunking of the four motor programs originally involved.

Given the theoretical relevance of Klapp's hypothesis, it would be desirable to re-evaluate its empirical evidence, in particular the one suggesting that a complete preparation of unfamiliar multiple-unit responses is not possible in advance. Indeed, the longer latency of the unfamiliar multipleunit responses than the unfamiliar single-unit responses, which Klapp (1) found in simple reaction time tasks could be simply due to the specific temporal relationship between the cue and the target he used. In his 1st study (Experiment 1), the cue-target interval varied randomly between 2000 and $2750 \mathrm{~ms}$, and in his 2nd study (Experiment 2), it varied randomly between 2000 and 2400 ms. It might be difficult for the observer to completely prepare untrained multiple-unit responses (but not single-unit responses) in advance, without certainty about their triggering moment. $A$ related possibility is that it might be difficult for the observer to maintain complete preparation of multiple-unit responses (but not single-unit responses) for hundreds of milliseconds until the appearance of the target.

The present study investigated in the 1st experiment whether untrained complex responses, putatively formed by multiple units, can be completely prepared in advance when the exact moment of their triggering is known with certainty. According to Klapp's hypothesis, complete preparation would not occur, even in this condition. To further test Klapp's hypothesis, we additionally investigated in the 2nd experiment the possibility of a complete preparation of the same complex responses after their extensive training. As this procedure tends to integrate the various units of the complex response into a single unit $(1,5)$, Klapp's hypothesis would predict their complete preparation in advance.

\section{Experiment 1}

This experiment examined the possibility of a complete advance preparation of untrained complex responses, whose spatial-temporal pattern and possible moments of triggering were signaled by a visual cue.

A simple response task, in which the same one-submovement response was required in all trials, and a complex response task, in which different four-submovement responses were required in different trials, were used to test the participants. The target for triggering these responses was the same in both tasks. A cue, which in the simple response task provided probabilistic temporal information about the moment of response triggering and in the complex response task, the same probabilistic temporal information and also information about the spatial-temporal pattern of the response, preceded the target by a short, a medium or a long interval.

For the simple response task and, more particularly, for the complex response task, reaction time was expected to decrease with the increase in cue-target interval, since there would be more time for advance response preparation and more certainty about the exact moment of response triggering. The long cue-target interval is particularly instructive since it would be enough for complete preparation of the simple response and, presumably, also of the complex responses, and would provide information about the moment of triggering of these responses with absolute certainty. If a longer latency of the complex responses than the simple response is found using this interval, one should conclude that a complete advance preparation of complex responses is in fact not possible. If instead the latency of the complex responses becomes similar to that of the simple response using this interval, the conclusion that this preparation is perfectly possible, depending only on the existence of an adequate probabilistic temporal relationship between the cue and the target, would be more appropriate.

Unilateral and bilateral responses as well as left and right hand responses were tested to allow the generalization of the conclusions of the study to these different motor conditions. Submovement and pause (intersubmovement) times were also evaluated to detect possible changes in motor executive processes related to different degrees of complex response preparation. This might help the interpretation of the reaction time results in addition to providing interesting information on its own.

\section{Material and Methods}

\section{Participants}

Twelve healthy undergraduate students from the University of São Paulo (6 males) were tested. Their ages ranged from 18 to 23 years. All were right-handed according to the Edinburgh Inventory (6) and had normal or correctedto-normal vision. All were naive regarding the purpose of the experiment.

The Research Ethics Committee of the Instituto de Ciências Biomédicas, Universidade de São Paulo, approved this study and written informed consent was obtained from all participants.

\section{Apparatus}

The participants were tested individually in a dimly illuminated and sound-attenuated room. They remained seated in front of a table, with their head positioned in a chin-and-front rest. Their eyes were at the same level and $57 \mathrm{~cm}$ away from the center of a $49-\mathrm{cm}$ wide and $46-\mathrm{cm}$ high blackboard. At this location was a red light-emitting diode (LED) whose flashing was used as the target stimulus. Two degrees above this LED was a row of three other red LEDs, the middle one located at the midline and the lateral ones located 2 degrees from it (see Figure 1). The flashing of these LEDs was used as the cue for the response. The presentation of the cue and of the target was controlled by a computer and programs developed in the MEL2 Professional 
(Psychology Software Tools Inc., USA) environment.

Two pairs of optic switch keys were fixed on the table, one on its right side and one on its left side, $22 \mathrm{~cm}$ far from the midline. These keys served to record the responses of the participants and were connected to a custom-made coupler unit.

Signals from the parallel port of the computer controlling the LEDs and from the key coupler unit were sampled at a rate of $2000 \mathrm{~Hz}$ per channel by means of a 1401 plus analog-digital converter (Cambridge Electronic Design Ltd., UK) and stored in a second computer. The Spike 2 software (Cambridge Electronic Design Ltd.) was used to acquire the data. Programs written in the Spike 2 script language environment were used to calculate the latency of the response, and the durations of the submovements and pauses between them, in each trial.

\section{Procedure}

Two testing sessions were conducted, one in which the participant performed a simple response task and the other in which the participant performed a complex response task. Before these testing sessions, the participant read a simplified description of the required task. Next, the participant was shown the stimuli and response keys. The testing sessions were composed of three blocks of 30 trials. Immediately before each one of these blocks, the participant was informed about how to respond and was then asked to perform some practice trials; next the participant performed all the trials of the block.

In the simple response task, the participant was instructed to keep the eyes on the central LED bulb, observe the flashing of the left, the right or the left plus right upper LEDs (cue) and respond by the flashing of the central LED

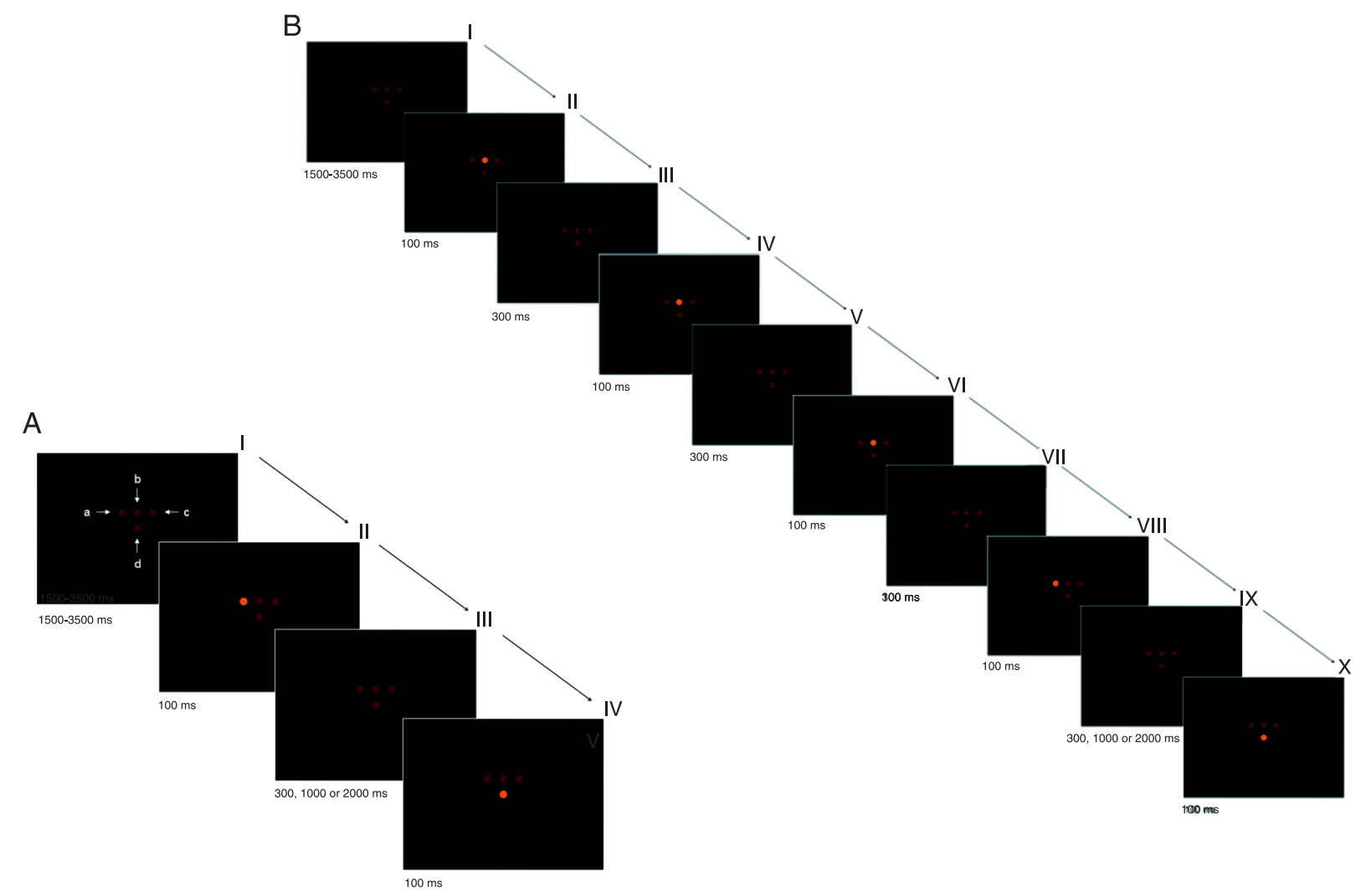

Figure 1. Schematic representation of a trial of the simple response task ( $P$ anel $A)$ and a trial of the complex response task $(P a n e l B)$ in Experiments 1 and 2. The letters a, b, and c indicate the left, middle and right upper light-emitting diodes (LEDs), respectively; the letter $d$ indicates the central LED. In Panel A, frame I shows the display during the variable interval between the beginning of the trial and the appearance of the cue, frame II shows the cue display, frame III shows the display during the variable interval between the cue and the target, and frame IV shows the target display. In Panel B, frame I shows the display during the variable interval between the beginning of the trial and the appearance of the cue, frames II to VIII show one of the 10 possible cue displays, frame IX shows the display during the variable interval between the cue and the target, and frame X shows the target display. The cue and the target lasted $100 \mathrm{~ms}$. Cue-target interval could be 300, 1000, or $2000 \mathrm{~ms}$. 
(target; see Figure 1A). Cue and target flashes lasted $100 \mathrm{~ms}$, and the interval between these stimuli (cuetarget interval, CTI) could be 300, 1000, or 2000 ms. In one block of trials, the cue was the flashing of the left upper LED and the participant was required to release and again press the external left key with the left middle finger. In another block of trials, the cue was the flashing of the right upper LED and the participant was required to release and again press the external right key with the right middle finger. In another block of trials, the cue was the flashing of the left plus right upper LEDs and the participant was required to release and again press the left and right external keys with the left and right middle fingers, respectively. Each CTI occurred randomly 10 times during each of these blocks.

In the complex response task the participant was instructed to to keep the eyes on on the central LED bulb, observe the spatial sequence of flashing of the left and middle, the right and middle, or the left plus right and middle upper LEDs (cue), and respond according to this sequence by the flashing of the central LED (target; see Figure 1B). Each cue consisted of four flashes, which lasted $100 \mathrm{~ms}$ and were separated by an interval of 300 $\mathrm{ms}$. These four flashes could occur in 10 different spatial sequences. As in the simple response task, the target lasted $100 \mathrm{~ms}$ and CTI could be 300, 1000, or $2000 \mathrm{~ms}$. In the block of trials in which the left and the middle upper LED flashed, the participant was required to move the left middle finger so as to replicate the spatial sequence of flashing of these LEDs (the left upper LED flashing indicated that the left external key should be pressed and the middle upper LED flashing indicated that the left internal key should be pressed). In the block of trials in which the right and middle upper LED flashed, the participant was required to move the right middle finger so as to replicate the spatial sequence of flashing of these LEDs (the right upper LED flashing indicated that the right external key should be pressed and the middle upper LED flashing indicated that the right internal key should be pressed). In the block of trials in which the left plus right upper LEDs and the middle upper LED flashed, the participant was required to move the left and right middle fingers so as to replicate the spatial sequence of flashing of these LEDs (the left plus right upper LEDs flashing indicated that both the left and right external keys should be pressed and the middle upper LED flashing indicated that both the left and right internal keys should be pressed). The initial position of the middle fingers in all trials was pressing the external keys. All the responses began, then, by the release of one or of both these keys. They included inwards, outwards and/or up-and-down submovements.

The testing order of the left, right and left-right sides in the session, as well as the testing order of the simple and complex response tasks were randomized among the participants.

\section{Statistical analysis}

Reaction time (interval between flashing of the target and lifting of the finger from the external key), submovement times (interval between releasing a key and pressing the same or the other key of the pair) and pause times (interval between pressing and releasing a given key) were automatically measured by means of Spike 2 script language routines. For each condition, the median of each parameter (reaction time, 1st submovement time, 1st pause time, 2nd submovement time, 2nd pause time, 3rd submovement time, 3rd pause time, and 4th submovement time) was obtained after excluding reaction times below 150 or above $2000 \mathrm{~ms}$, submovement and pause times lasting less than 50 or more than $150 \mathrm{~ms}$ and times of submovements not indicated by the cue.

Reaction time, submovement times and pause times were separately submitted to a repeated measures analysis of variance. In the case of the reaction time and the 1st submovement time, factors in these analyses were the task (simple response or complex response), the interval between the cue and the target $(300,1000$, or $2000 \mathrm{~ms})$, the responding mode (unilateral or bilateral) and the responding hand (left or right). In the case of the 2nd, 3rd, and 4th submovement times and the 1st, 2nd, and 3rd pause times, factors in these analyses were the interval between the cue and the target $(300,1000$, or $2000 \mathrm{~ms})$, the responding mode (unilateral or bilateral) and the responding hand (left or right). When appropriate, the data were further analyzed by the post hoc Tukey test. The level of significance of 0.05 was adopted for all analyses.

\section{Results and Discussion}

The results obtained with each parameter will be described separately.

\section{Reaction time}

ANOVA showed a main effect of task $(F 1,11=72.71 ; P$ $<0.001)$ and CTI $(F 2,22=51.22 ; P<0.001)$ and an interaction between task and CTI (F2,22 = 52.42; $\mathrm{P}<0.001)$. Reaction time was longer for the complex response task than for the simple response task for the three CTI (in all cases, $\mathrm{P}<0.001)$. For the simple response task, reaction times did not differ between the three CTI. For the complex response task, reaction time was shorter for the 1000- and the 2000-ms CTI than for the 300-ms CTI (in both cases, $P<0.001$; Figure 2A).

The similar latency of the previously untrained simple response by the three CTI was unexpected considering the very different probabilities of response triggering by them ( $30 \%$ by the $300-\mathrm{ms} \mathrm{CTI,} 50 \%$ by the $1000-\mathrm{ms}$ CTI and $100 \%$ by the $2000-\mathrm{ms} \mathrm{CTI}$ ). The finding suggests that the strategy of preparing the response and then maintaining this preparation until the next interval was adopted or, more likely, preparing the response a 1 st time by one interval and 
then preparing it again by the next interval (see Ref. 7, for a discussion about these two strategies).

The large decrease in the latency of the untrained complex responses from the 300 - to the 1000 -ms CTI should be ascribed mainly to the possibility of completing the rehearsal of the cue pattern and advancing motor preparation before the appearance of the target. The fact that no further latency reduction occurred from the 1000 - to the 2000-ms CTI suggests that the maximum preparation, which was possible in advance was achieved in less than $1000 \mathrm{~ms}$ and that the strategy of maintaining response preparation or preparing it anew, presumably adopted in the simple response task, was also used in the complex response task.

The longer latency of the cued untrained complex responses than the cued previously untrained simple response replicates previous results in the literature (1-4,8). The observation that by both the 1000- and the 2000-ms $\mathrm{CTI}$, when maximum preparation of the untrained complex responses occurred, the latency of these responses was longer than the latency of the previously untrained simple response confirms and extends the data reported by Klapp $(1,2)$. It also reinforces his proposal that the preparation of multi-unit motor responses can only be completed after their triggering.

\section{Submovement times}

ANOVA showed a main effect of task $(F 1,11=47.45 ; P$ $<0.001)$ and CTI (F2,22 = 4.41; P < 0.025) and an interac-
A
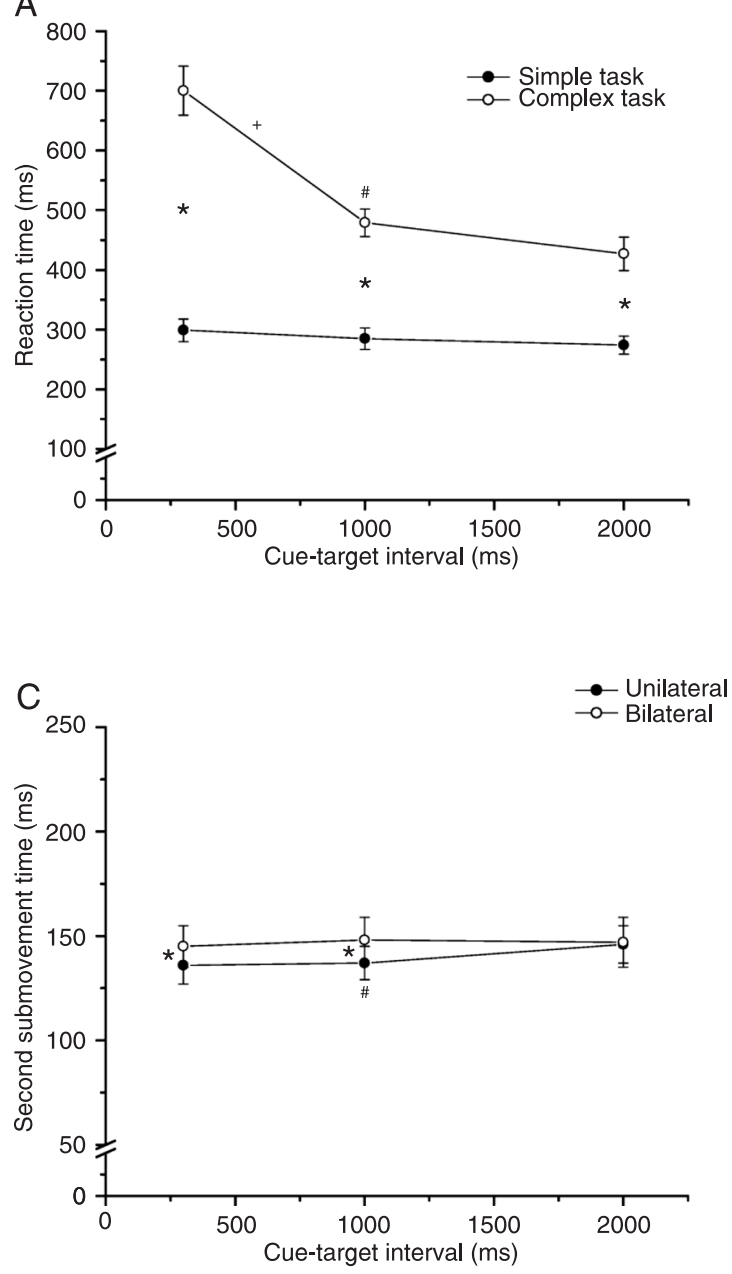
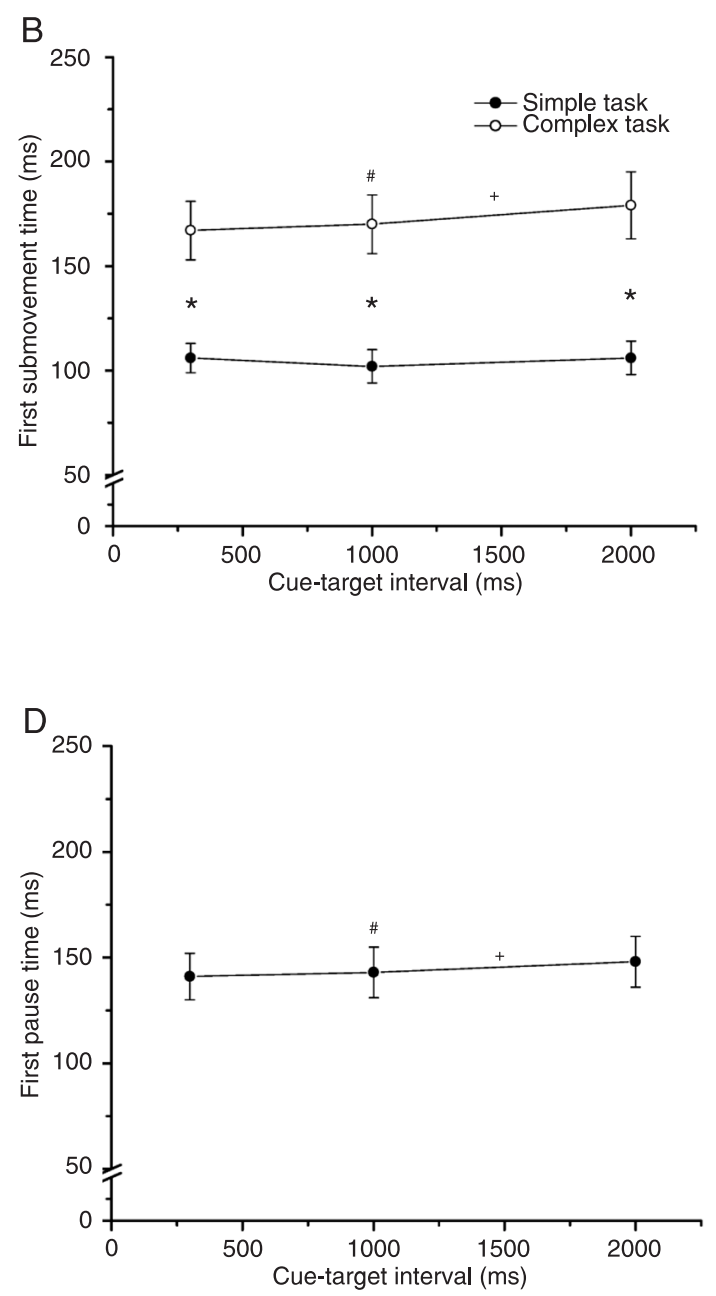

Figure 2. Reaction time $(A)$ and 1 st submovement time $(B)$ obtained in Experiment 1 for each cue-target interval in the simple and complex response tasks. Second submovement time $(C)$ and 1 st pause time $(D)$ obtained in Experiment 1 for each cue-target interval in the complex response task. Data are reported as means \pm SEM $(N=12)$. ${ }^{*} P<0.05$ for the difference between the simple and complex task or between the unilateral and the bilateral responding mode; ${ }^{+} \mathrm{P}<0.05$ for the difference between the 300 - and $1000-\mathrm{ms}$ cue-target interval or the 1000- and the 2000-ms cue-target interval; \#P $<0.05$ for the difference between the 300- and the 2000-ms cue-target interval (repeated measures analysis of variance and Tukey test). 
tion between task and CTI (F2,22 = 6.03; $\mathrm{P}=0.008)$ for the 1st submovement time. This submovement time was longer for the complex response task than for the simple response task for the three CTI (in all cases, $\mathrm{P}<0.001$ ). For the simple response task, 1st submovement times did not differ between the three CTI. For the complex response task, 1st submovement time was longer for the 2000-ms CTI than for the 300- and the 1000-ms CTI ( $P=0.001$ and $P=0.020$, respectively; Figure $2 B$ ).

For the 2nd submovement time, ANOVA showed a main effect of $\mathrm{CTI}(\mathrm{F} 2,22=4.22 ; \mathrm{P}=0.028)$ and an interaction between $\mathrm{CTI}$ and responding mode $(\mathrm{F} 2,22=3.58 ; \mathrm{P}=0.045)$. This submovement time was longer for the 2000-ms CTI than for the $300-\mathrm{ms} \mathrm{CTI}(P=0.009)$ for the unilateral mode. It was longer for the bilateral mode than for the unilateral mode for both the 300- and 1000-ms CTI ( $P=0.023$ and $\mathrm{P}=0.005$, respectively; Figure $2 \mathrm{C}$ ).

For the 3rd and 4th submovement times, ANOVA did not show any main effect or interaction.

An additional planned ANOVA, having as factors the order of the submovement (1st, 2nd, 3rd, or 4th) and the $\mathrm{CTI}$, showed a main effect of order $(\mathrm{F} 3,33=16.18 ; \mathrm{P}<$ $0.001)$, CTI (F2,22 = 3.61, P = 0.044) and an interaction between the two factors $(F 6,66=2.80 ; P=0.017)$. The 2nd, $3 \mathrm{rd}$, and 4th submovement times were shorter than the 1st submovement time for the 300-, 1000-, and 2000-ms CTI (in all cases, $\mathrm{P}<0.001$ ).

The longer duration of the 1st submovement of the untrained complex responses than the submovement of the previously untrained simple response suggests that there was some programming during its execution. This possibility is further supported by the duration of the 1st submovement being also longer than the duration of the subsequent submovements. As there was no difference between the durations of the $2 \mathrm{nd}$, 3rd, and 4th submovements, any programming eventually occurring while they were being executed should be quite similar.

The increase of the duration of the 1st submovement and the unilateral 2 nd submovement of the untrained complex responses from the 300 - to the $2000-\mathrm{ms}$ CTI might be explained by an increase of online programming possibly related to the degree of certainty about the moment of target appearance. This would allow earlier responding.

\section{Pause times}

ANOVA showed a main effect of interval $(F 2,22=7.82 ; \mathrm{P}$ $=0.003)$ for the $1 \mathrm{st}$ pause time. This pause time was longer for the 2000-ms CTI than for the 300- and 1000-ms CTI (P $=0.002$ and $P=0.034$, respectively; Figure 2D).

For the 2nd and 3rd pause times, ANOVA did not show any main effect or interaction.

An additional planned ANOVA, having as factors the order of the pause (1st, 2nd or 3rd) and the CTI, showed a main effect of order (F2,22 $=4.97 ; \mathrm{P}=0.017)$ and $\mathrm{CTI}$ $(F 2,22=5.12 ; P=0.015)$. The 3rd pause time was shorter than the 1st $(P=0.015)$.

The longer duration of the 1 st pause than of the $2 \mathrm{nd}$ and 3 rd pauses and its increase from the 300- to the 2000-ms CTI paralleled the results obtained for the duration of the 1 st submovement. As was the case for this submovement, these changes should have been produced by online programming of the response.

\section{Experiment 2}

According to Klapp (1), trained complex responses, differently from untrained complex responses, can be prepared completely in advance due to the integration along the practice trials of the programs controlling the individual submovements of the response. This experiment examined the possibility that the complex responses tested in Experiment 1 could be prepared completely in advance after their extensive training.

As in Experiment 1, the participants performed a simple response task and a complex response task. The responses to be performed in these tasks were now extensively trained before the testing sessions. In the simple response task, the participants performed in all trials of the testing session the same one-submovement response. In the complex response task, the participants performed in all trials of the testing session the particular four-submovement response, which they had trained. The target for triggering these responses was the same in both tasks. The cue, which preceded the target by the short, the medium or the long CTI, provided basically probabilistic information about the moment of response triggering in both tasks.

Taking into account the result obtained in Experiment 1 , similar reaction times were expected for the three CTI in the simple response task. In the complex response task, reaction time was expected to decrease from the short to the medium CTI since trained complex response preparation should take more than $300 \mathrm{~ms}$. The latency of the trained complex responses might become similar to the latency of the trained simple response by the medium or at least by the long CTI. Such a result would indicate the occurrence of a complete advance preparation of the trained complex responses; in addition, it would give a gross indication of the time required for this preparation to occur.

As in Experiment 1, unilateral and bilateral responses as well as left and right hand responses were tested, and submovement and pause times were evaluated.

\section{Material and Methods}

\section{Participants}

Twelve healthy undergraduate students from the University of São Paulo (6 males) were tested. Their ages ranged from 18 to 26 years. All were right-handed and had normal or corrected-to-normal vision. All were naive regarding the purpose of the experiment. 


\section{Apparatus}

The apparatus described in Experiment 1 was used.

\section{Procedure}

Three testing sessions were conducted. In the 1st one, the participant was trained to perform a sequence of four submovements (the spatial sequences of four submovements used in Experiment 1 were randomly distributed to the participants; since there were 10 spatial sequences and 12 participants, two of the spatial sequences were distributed to two participants). This session had three blocks of trials. In the 1st block, the left upper LED and the middle upper LED flashings were used as cue and the participants reacted with their left hand; in the 2 nd block, the right upper LED and the middle upper LED flashings were used as cue and the participants reacted with their right hand, and in the 3rd block the left plus right upper LEDs and the middle upper LED flashings were used as cue and the participants reacted with both hands.

In the 2nd testing session, the participants performed the simple response task used in Experiment 1, and in the 3rd, they performed the complex response task practiced in the 1 st testing session. With the exception of sequence constancy for each participant in the complex response task, the stimulatory and response conditions in these two tasks were the same as those described in Experiment 1.

\section{Data analysis}

Reaction time, submovement times and pause times were obtained and the median of these parameters was calculated for each condition. The data obtained were submitted to repeated measures ANOVA and the Tukey test as described for Experiment 1.

\section{Results and Discussion}

The results obtained with each parameter will be described separately.

\section{Reaction time}

ANOVA showed a main effect of task $(F 1,11=6.86$; $P$ $=0.024)$ and CTI (F2,22 = 42.83; P < 0.001) and an interaction between task and CTI (F2,22 = 15.69; $\mathrm{P}<0.001)$. Reaction time was longer for the complex response task than for the simple response task for the 300- and the 1000ms CTI ( $P<0.001$ and $P=0.033$, respectively) but not for the 2000-ms CTI. For the simple response task, reaction times did not differ between the three CTI. For the complex response task, reaction time was shorter for the 1000- and the 2000-ms CTI than for the 300-ms CTI (in both cases, $P<0.001$; Figure 3A).

The longer latency of the trained complex responses than the trained simple response observed for the 300- and 1000-ms CTI replicated the results obtained for these CTI in the previous experiment. However, the latency differences were now smaller than before. For the 2000-ms CTI, the latencies of the trained complex responses and the trained simple response were fairly similar, contrasting with the important difference observed in that experiment.

The closer latencies of the trained complex responses and the trained simple response in the current experiment, culminating with equivalency for the 2000-ms CTI, should be credited initially to the fact that no rehearsal of the cue pattern signaling the former responses would presumably have been necessary. In addition, preparation of the trained complex responses should have become faster due to the formation of a single motor program or motor "chunk" $(1,2)$ representing their four submovements. This would have made the preparation of these responses more similar to that of the simple response.

It should be noted, however, that the mechanisms controlling the trained complex responses, even putatively formed by a single motor program, still needed more time to be fully organized than those controlling the trained simple response. This is indicated by the longer trained complex response latencies observed for the 300- and 1000-ms CTI.

\section{Submovement times}

ANOVA showed a main effect of CTI (F2,22 = 17.23; $P$ $<0.001$ ) for the 1 st submovement time, which was longer for the 2000-ms CTI than for the 300-and the 1000-ms CTI $(P<0.001$ and $P=0.002$, respectively; Figure 3B).

For the 2nd submovement time, ANOVA showed a main effect of CTI (F2,22 = 7.20; P = 0.004). This submovement time was longer for the 1000- and 2000-ms CTI than for the 300-ms CTI ( $P=0.032$ and $P=0.004$, respectively; Figure 3C).

For the 3rd and 4th submovement times, ANOVA did not show any main effect or interaction.

An additional planned ANOVA, having as factors the order of the submovement (1st, $2 \mathrm{nd}$, 3rd, or 4th) and the CTI, showed a main effect of CTI $(F 2,22=11.17, \mathrm{P}<0.001)$ and an interaction between the two factors (F6,66 $=5.71, P$ $<0.001$ ). The 2 nd submovement time was shorter than the 1st submovement time for the 300- and the 2000-ms CTI $(P=0.001$ and $P<0.001$, respectively). The 3rd submovement time was shorter than the 1 st and 2 nd submovement times for the three CTI (in all cases $P<0.001$ ). The 4th submovement time was shorter than the 1 st submovement time for the three CTI (in all cases $\mathrm{P}<0.001$ ) and the 2nd submovement time for the 1000- and the 2000-ms CTI (in both cases $P<0.001$ ), and was longer than the 3rd submovement time for the three CTI $(P<0.001, P<0.001$, and $P=0.001$, respectively).

The increase of the duration of the submovement of the trained simple response from the 300 - to the $2000-\mathrm{ms}$ CTI distinguishes this response from the previously untrained simple response in the previous experiment. The increase of the duration of the 1 st and 2 nd submovements of the 
trained complex responses from the 300 - to the $2000-\mathrm{ms}$ CTI reproduced the results obtained for the untrained complex responses in the previous experiment. Both results can be explained by an increase of online response programming. As considered before, this might be related to the degree of certainty about the moment of target appearance.

\section{Pause times}

ANOVA showed a main effect of responding hand $(F 1,11=14.78 ; P=0.003)$ and an interaction between CTI and responding mode $(F 2,22=4.72 ; \mathrm{P}=0.020)$ for the 1st pause time. This pause time was shorter for the right hand than for the left hand. For the unilateral mode, it was longer for the 2000-ms CTI than for the 300-ms CTI ( $P=$ 0.044; Figure 3D).

A

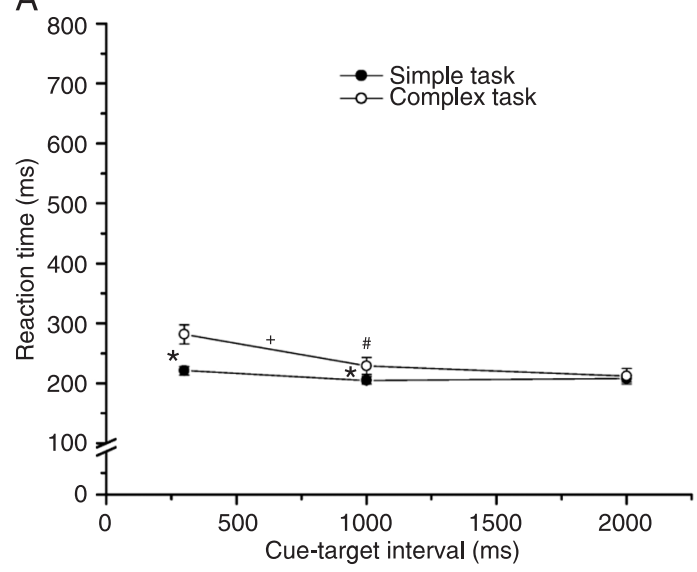

C

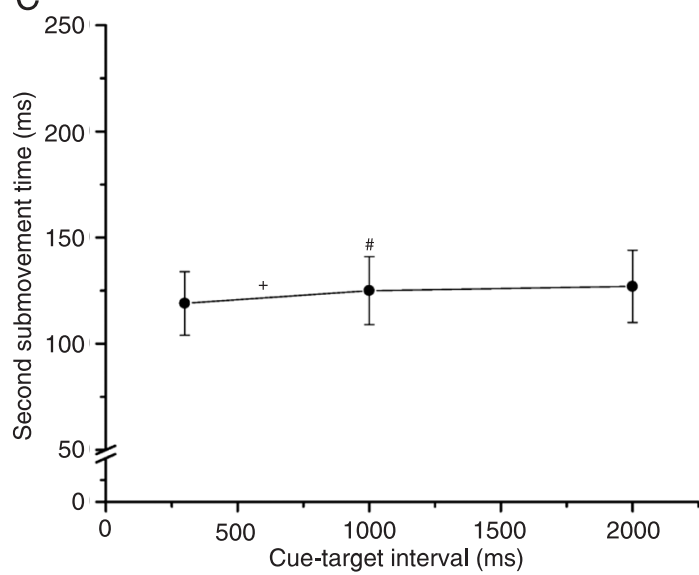

For the 2nd pause time, ANOVA did not show any main effect or interaction.

For the 3rd pause time, ANOVA showed a main effect of responding hand $(F 1,11=5.30 ; P=0.042)$. This pause time was shorter for the right hand than for the left hand.

An additional planned ANOVA, having as factors the order of the pause (1st, 2nd or 3rd) and the CTI, showed no main effect or interaction.

The increase of the duration of the unilateral 1st pause of the trained complex responses from the 300- to the 2000ms CTI reproduced the result obtained for the untrained complex responses in the previous experiment. As suggested for the increase of the duration of the 1st pause of these responses, it should have been produced by online programming of the response.
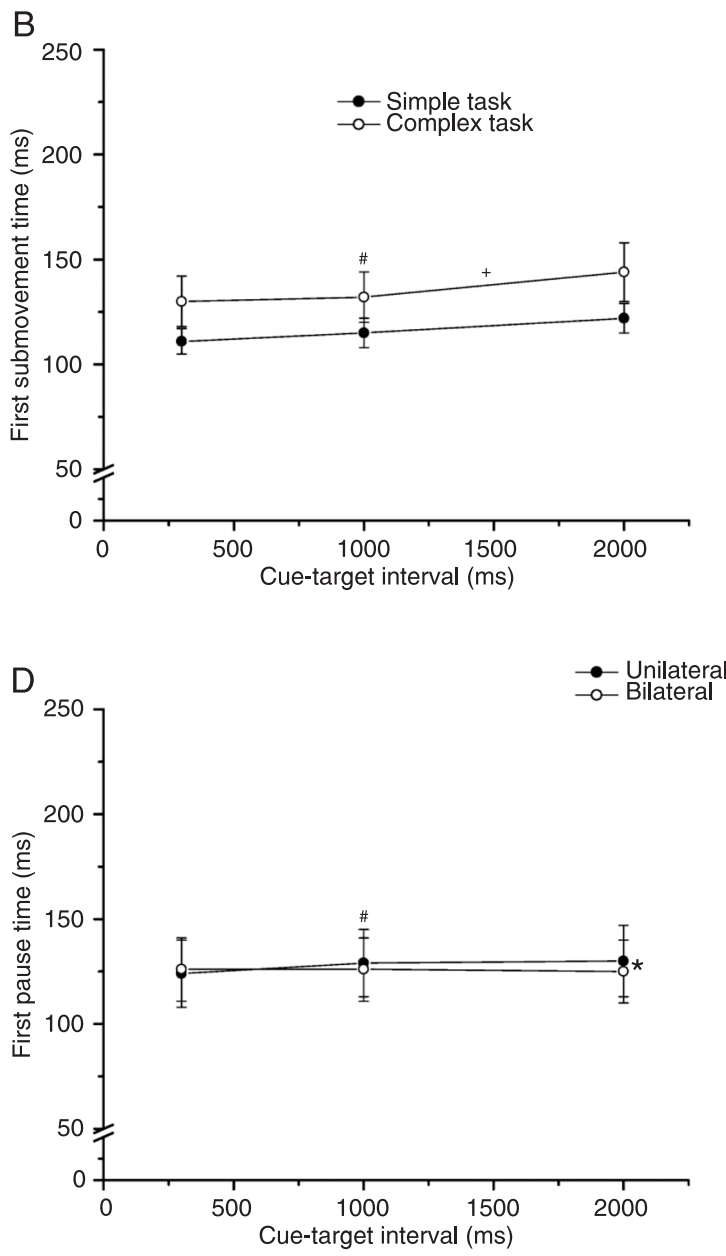

Figure 3. Reaction time $(A)$ and 1st submovement time $(B)$ obtained in Experiment 2 for each cue-target interval in the simple and complex response tasks. Second submovement time $(C)$ and 1 st pause time $(D)$ obtained in Experiment 2 for each cue-target interval in the complex response task. Data are reported as means \pm SEM $(N=12)$. ${ }^{*} P<0.05$ for the difference between the simple and the complex task or between the unilateral and bilateral responding mode; ${ }^{+} \mathrm{P}<0.05$ for the difference between the 300 - and 1000 -ms cue-target interval or the 1000- and the 2000-ms cue-target interval; ${ }^{*} P<0.05$ for the difference between the 300- and 2000-ms cuetarget interval (repeated measures analysis of variance and Tukey test). 
The shorter duration of the 1st and 3rd pauses of the right hand than of the left hand in the present experiment suggests that practice increases more the efficiency of the left hemisphere digit control mechanisms than the efficiency of the right hemisphere digit control mechanisms. This hypothesis deserves to be further investigated.

\section{General Discussion}

The main goal of the present study was to determine whether cued untrained complex responses can be prepared in advance as completely as a cued previously untrained simple response when enough time is provided for this preparation to occur and there is certainty about the moment of response triggering. It was demonstrated that the latency of the tested untrained complex responses was longer than the latency of a previously untrained simple response by the three cue-target intervals used, namely, 300 , 1000 , and $2000 \mathrm{~ms}$. The latency of the untrained complex responses decreased from the short to the medium CTI but did not further change with the long CTI, suggesting that maximum possible preparation was achieved. These results indicate that untrained complex responses can be partially prepared in advance. However, complete preparation of these responses is not possible, even when favored by a relatively long $\mathrm{CTI}$ and absolute certainty about the moment of response triggering. These conclusions are in agreement with those reached by Klapp $(1,2)$ on the basis of somewhat less conclusive evidence.

The advance preparation of untrained complex responses would involve the organization of the internal structure of the 1st unit of the response ("chunk") and the activation and scanning of the abstract time frame, which specifies the time of initiation of each one of the units ("chunks") of the response, as proposed by Klapp (2). The alternative to these processes suggested by Magnuson et al. (8), namely the compilation of all the units of the response and the forwarding from long-term memory to a hypothetical motor buffer of a template, which would specify the response unit sequence, seems less appropriate. As pointed out by Klapp and Jagacinski (9), it requires the assumption that there is a compilation of every unit of the response even when all units are identical and, more critically, it is not compatible with the mutual exclusivity principle of Gestalt applied to the motor system, which predicts the impossibility of organizing more than one response unit ("chunk") at a time.

The present findings support Klapp's hypothesis that multiple-unit response preparation involves a critical component, which can only be mobilized by the moment of response triggering. Whether this component is represented by the activation and scanning of an abstract time frame, which would specify the time of initiation of each unit of the response, as proposed by Klapp (2, see also Ref. 9), remains to be proved.

The current study also demonstrated that the latency of the trained complex responses was longer than that of the previously trained simple response by the 300- and 1000-ms cue-target intervals but became similar to it by the 2000-ms cue-target interval. These results indicate that a complete preparation of trained complex responses can occur in advance but that this takes a reasonable time. Klapp (1) had already shown that trained complex responses could be completely prepared in advance. However, the relatively long time required for this preparation was not previously demonstrated.

Practice is believed to lead to a restructuration of complex response programming $(1,5,10,11)$. Part of this restructuration would involve the integration of the motor programs controlling the different units of a multiple-unit response so as to form a few or even a single larger motor program $(1,2,5)$. If the trained complex responses of the current study became controlled by a single motor program, their preparation would involve only the organization of the internal structure of this program; the putative activation and scanning of an abstract time frame responsible for specifying the time of initiation of the units of the response would not be required. As the former process could develop completely before the appearance of the target, complete advance preparation of the trained complex responses would have been possible.

The longer time necessary to complete the preparation of the trained complex responses than the trained simple response should be attributed to the greater complexity of their controlling motor program, which would include the neural networks specifying each one of their four submovements.

The observed shorter latency of the trained responses than the untrained responses is not a surprise. It is also not a surprise the fact that this difference was larger for the complex responses. These results replicate findings in the literature $(1,2,8,12)$. They reinforce the idea that practice induces a large restructuration of response programming, which makes it much more efficient. Understandably, this change benefits more complex responses than simple responses since there is more room for improvement in the case of the former responses.

In addition to the reaction time results, the current study showed that the duration of the 1st submovement of the untrained complex responses was longer than the duration of the $1 \mathrm{st}$ submovement of the previously untrained simple response and that the duration of the $2 \mathrm{nd}$, 3rd, and 4th submovements was shorter than the duration of the $1 \mathrm{st}$ submovement for both the untrained and trained complex responses. As already discussed, these results suggest the occurrence of online programming by the initial part of the complex responses, more for the untrained than for the trained complex responses.

A very interesting observation was the increase of the duration of the 1 st submovement, 1st pause and 2nd submovement of both the untrained and trained complex 
responses from the $300-$ to the $2000-\mathrm{ms}$ CTI. This robust result is original in the literature and suggests the occurrence of partial online programming of the complex responses and that this online programming increased with the certainty about the moment of appearance of the target. Possibly, complex response preparation would involve not only the organization of the internal structure of the response and ordering of its units, as proposed by Klapp $(1,2)$, but also the transfer of part of the programming of the response to its execution stage.

In addition to the increased duration between the short and long CTI, shared with the 1st pause and 2nd submovement, the 1 st submovement showed a clear duration increase between the medium and the long CTI. This was observed for both the untrained and trained complex responses. Although some extra online programming could be the reason for this particular finding, it is tempting to speculate whether it was related to some inhibition of motor executive processes during response preparation to avoid anticipated responding. This kind of error is common when reaction speed is emphasized and the moment of appearance of the target is certain. The hypothesis is made particularly attractive on the basis of the existence of electrophysiological evidence indicating that the motor execution mechanisms are inhibited during response preparation (13-19, see also Ref. 20). The similar increased duration of the submovement of the trained simple response between the medium and the long CTI could be seen as strengthening the hypothesis, if it is accepted that the modest online programming expected for this kind of response would not be able alone to account for the finding.

The obvious decrease of the submovement and pause times with practice should have been caused, at least in part, by a reduction of online programming of the complex responses. This would be in agreement with the hypothesis of a more extensive preparation of these responses after

\section{References}

1. Klapp ST. Motor response programming during simple and choice reaction time: the role of practice. J Exp Psychol: Hum Perc Perform 1995; 21: 1015-1027.

2. Klapp ST. Reaction time analysis of two types of motor preparation for speech articulation: action as a sequence of chunks. J Mot Behav 2003; 35: 135-150.

3. Khan MA, Lawrence GP, Buckolz E, Franks IM. Programming strategies for rapid aiming movements under simple and choice reaction time conditions. Q J Exp Psychol 2006; 59: 524-542.

4. Verwey WB. Processing modes and parallel processors in producing familiar keying sequences. Psychol Res 2003; 67: 106-122.

5. Khan MA, Mourton S, Buckolz E, Franks IM. The influence of advance information on the response complexity effect in manual aiming movements. Acta Psychol 2008; 127: 154- practice (1). Another factor, which might have contributed to reducing submovement and pause times, would be a practice-induced integration and/or parallel activation of the neural operations involved in controlling response execution. This would increase the efficiency of this process $(1,2,10)$. It would be interesting to specifically investigate the relative importance, if any, of this latter factor for fast response execution.

It is interesting to note that the reduction of the duration of the 1st submovement of the complex responses with practice was much less than the reduction of the latency of these responses. This argues against the possibility that the latter result was due to the adoption of the strategy of partially programming the movements during their execution and not to a more complete response preparation. This alternative was considered by Klapp (1) to explain results similar to the present ones, and was equally rejected by him.

In conclusion, the present study showed that cued untrained complex responses cannot be completely prepared in advance even when there is time enough for this preparation to occur and the moment of response triggering is known with certainty, and that cued trained complex responses can be completely prepared in advance although requiring an appreciable time for that. This study further showed that the duration of the initial components of untrained and trained complex responses increases with cue-target interval, suggesting an increase of online response programming related to the certainty about the moment of target appearance.

\section{Acknowledgments}

S.R. Alouche was the recipient of a CAPES doctoral scholarship and G.N. Sant'Anna was the recipient of a CNPq-PIBIC scholarship.
162.

6. Oldfield RC. The assessment and analysis of handedness: the Edinburgh Inventory. Neuropsychologia 1971; 9: 97113.

7. Niemi $P$, Näätänen R. Foreperiod and simple reaction time. Psychol Bull 1981; 89: 133-162.

8. Magnuson CE, Robin DA, Wright DL. Motor programming when sequencing multiple elements of the same duration. $J$ Mot Behav 2008; 40: 532-544.

9. Klapp ST, Jagacinski RJ. Gestalt principles in the control of motor action. Psychol Bull 2011; 137: 443-462.

10. Smiley-Oyen AL, Worringham CJ. Distribution of programming in a rapid aimed sequential movement. $Q J$ Exp Psychol A 1996; 49: 379-397.

11. Verwey WB, Eikelboom T. Evidence for lasting sequence segmentation in the discrete sequence-production task. $J$ 
Mot Behav 2003; 35: 171-181.

12. Rhodes BJ, Bullock D, Verwey WB, Averbeck BB, Page MP. Learning and production of movement sequences: behavioral, neurophysiological, and modeling perspectives. Hum Mov Sci 2004; 23: 699-746.

13. Mojica JA, Yamada Y, Nakamura R. Effect of warning signal on reaction time and EMG activity of the biceps brachii muscle in elbow flexion and forearm supination. Tohoku $J$ Exp Med 1988; 154: 375-380.

14. Touge T, Taylor JL, Rothwell JC. Reduced excitability of the cortico-spinal system during the warning period of a reaction time task. Electroencephalogr Clin Neurophysiol 1998; 109: 489-495.

15. Hasbroucq T, Kaneko H, Akamatsu M, Possamai CA. The time-course of preparatory spinal and cortico-spinal inhibition: an H-reflex and transcranial magnetic stimulation study in man. Exp Brain Res 1999; 124: 33-41.

16. Takei T, Hashimoto T, Hagura N, Matsumura M, Naito E.
Reduction of cortico-spinal excitability by transcranial magnetic stimulation at predictable timing. Jpn J Physiol 2005; 55: 93-99.

17. Duclos Y, Schmied A, Burle B, Burnet H, Rossi-Durand C. Anticipatory changes in human motoneuron discharge patterns during motor preparation. J Physiol 2008; 586: 10171028.

18. Sinclair C, Hammond GR. Reduced intracortical inhibition during the foreperiod of a warned reaction time task. Exp Brain Res 2008; 186: 385-392.

19. Sinclair C, Hammond GR. Excitatory and inhibitory processes in primary motor cortex during the foreperiod of a warned reaction time task are unrelated to response expectancy. Exp Brain Res 2009; 194: 103-113.

20. van den Hurk P, Mars RB, van Elswijk G, Hegeman J, Pasman JW, Bloem BR, et al. Online maintenance of sensory and motor representations: effects on corticospinal excitability. J Neurophysiol 2007; 97: 1642-1648. 